\title{
Programa de reabilitação de equilíbrio e marcha em pacientes com doença de Parkinson
}

\author{
Program for rehabilitation of balance and gait in patients with Parkinson's disease
}

Vanessa de Melo Dantas ${ }^{1}$ (D), Rafael de Oliveira Guiduce ${ }^{1}$ (D)

Marcos Henrique Dall'Aglio Foss ${ }^{1}$ (D), Lucas Lima Ferreira ${ }^{1}$

\begin{abstract}
RESUMO
Introdução: A doença de Parkinson (DP) é caracterizada por movimentos involuntários e alterações de equilíbrio e marcha que afetam as atividades de vida diária. Objetivo: Avaliar os efeitos de um programa de reabilitação de equilíbrio e marcha em pacientes com DP. Método: Estudo intervencional, realizado no Ambulatório de Fisioterapia de um hospital-escola, com indivíduos diagnosticados com DP. Foram coletados sexo, idade, tempo de diagnóstico, estágio da doença, segundo a escala de Hoehn \& Yahr, e medicações de uso regular. Os indivíduos participaram de um programa de reabilitação, duas vezes por semana, durante oito semanas. Avaliou-se o escore de equilíbrio e marcha por meio da escala de Tinetti antes e após a intervenção fisioterapêutica. Resultados: Foram incluídos 10 idosos, $60 \%$ do sexo masculino, média de idade de 66,6 \pm 10,75 anos, tempo médio de diagnóstico de 9,3 \pm 4,5 anos, estágio médio da doença $2,1 \pm 0,6$. Houve aumento significativo para o item levanta-se da cadeira $(p=0,05)$ e para o desequilíbrio no externo $(p=0,01)$ do teste de equilíbrio, na comparação antes e após a intervenção fisioterapêutica; também verificou-se aumento significativo no item comprimento e altura do passo $(p=0,005)$ e no escore total $(p=0,05)$ do teste de marcha. Na comparação do escore total da escala observouse incremento significativo $(\mathrm{p}=0,04)$ entre os momentos antes e após a intervenção realizada. Conclusão: O programa de reabilitação proposto promoveu melhora no equilíbrio e na marcha de um grupo de pacientes com DP.

Palavras-chave: equilíbrio postural; marcha; doença de Parkinson; modalidades de fisioterapia.
\end{abstract}

\begin{abstract}
Introduction: Parkinson's disease (PD) is characterized by involuntary movements and changes in balance and gait that affect the activities of daily living. Objective: To evaluate the effects of a balance and gait rehabilitation program in PD patients. Method: Interventional study, carried out in the outpatient clinic of a teaching hospital, with individuals diagnosed with PD. Sex, age, time since diagnosis, disease stage according to the Hoehn \& Yahr scale and regular use of medications were collected. The subjects participated in a rehabilitation program, twice a week for eight weeks. The balance and gait scores were assessed using the Tinetti scale before and after the physical therapy intervention. Results: Ten elderly people were included, $60 \%$ male, mean age $66.6 \pm 10.7$ years, mean time since diagnosis $9.3 \pm 4.5$ years, mean disease stage $2.1 \pm 0.6$. There was a significant increase for the item rises from the chair $(p=0.05)$ and for the external imbalance $(p=0.01)$ of the balance test in the comparison before and after the physical therapy intervention. A significant increase in the item length and height of the step $(\mathrm{p}=0.005)$ and in the total score $(\mathrm{p}=0.05)$ of the gait test was also verified. When comparing the total score of the scale, a significant increase $(\mathrm{p}=0.04)$ was observed between the moments before and after the intervention performed. Conclusion: The proposed rehabilitation program promoted an improvement in balance and gait in a group of patients with PD.
\end{abstract}

Keywords: postural balance; gait; Parkinson disease; physical therapy modalities.

\section{INTRODUÇÃO}

A doença de Parkinson (DP) é uma desordem neurodegenerativa progressiva que compromete os neurônios dopaminérgicos da substância negra. O déficit dessa substância implica em complicações das atividades motoras. ${ }^{1}$ Indivíduos com DP apresentam ao menos quatro sintomas específicos: tremores em repouso, lentidão na execução dos movimentos (bradicinesia), rigidez articular (hipertonia plástica), acometimento da musculatura flexora e alteração postural. Esta última está relacionada ao fato de que os portadores da DP tendem

${ }^{1}$ Faculdade de Medicina de Rio Preto - São José do Rio Preto (SP), Brasil.

Autor correspondente: Lucas Lima Ferreira - Faculdade de Medicina de São José do Rio Preto - Avenida Brigadeiro Faria Lima, 5.416 - Vila São Pedro - CEP: 15090-000 - São José do Rio Preto (SP), Brasil - E-mail: lucas_lim21 @ hotmail.com Recebido em 09/07/2020. Aceito para publicação em 14/12/2020. 
a ter postura fletida, cabeça anteriorizada, queixo em direção ao tórax, cifose torácica, ombros protraídos, braços rodados internamente, flexão de quadril, joelhos e cotovelos. ${ }^{1,2}$

A marcha também sofre alterações, tais como diminuição da amplitude dos movimentos nos membros inferiores, nos movimentos dos quadris, joelhos e tornozelos, bem como menor dissociação de cintura escapular, o que resulta na diminuição do comprimento dos passos e em dissociação da cintura pélvica. ${ }^{3}$ Os pacientes deambulam com uma marcha lentificada e arrastada, além de um persistente posicionamento da cabeça e do tronco para frente, deslocando, assim, o centro de gravidade, o que resulta em um padrão de marcha denominado "festinação"; atrelados a esse fator outros sintomas também são encontrados nesses pacientes: "freezing of gait", ou congelamento da marcha, caracteriza-se como um breve episódio que impede a ação da marcha devido à ausência ou à redução significativa de passos, mesmo com a intenção de executar o passo, e à dor presente decorrente da rigidez articular. ${ }^{4,5}$ Esses comprometimentos tendem a se agravar com a progressão da doença e a perda de massa muscular (sarcopenia), causada pelo avançar da idade. ${ }^{4}$

Estima-se que portadores da DP apresentem $62 \%$ mais risco de quedas, quando comparados a pacientes acometidos por outras doenças neurológicas. Com a perda dos reflexos posturais, portadores da DP tendem a não conseguir ficar em pé sem auxílio. ${ }^{5}$ Alguns estudos demonstram que as alterações no equilíbrio e na marcha são os principais fatores que geram incapacitantes para realização das atividades da vida diária e diminuição da qualidade de vida desses pacientes. ${ }^{6,7}$

A terapia farmacológica é a base do tratamento, sempre acompanhada e prescrita por um médico neurologista, porém a fisioterapia tem sua importância, pois promove exercícios que mantêm a musculatura preservada, agindo na mobilidade articular, na coordenação motora e na instabilidade postural, uma vez que atua em movimentos funcionais para promover diminuição dos sintomas ocasionados pela progressão da doença e prevenir deformidades que incapacitem esses indivíduos, além de promover convívio social e acolhimento pessoal. ${ }^{8-10} \mathrm{O}$ objetivo do presente estudo é avaliar os efeitos de um programa de reabilitação do equilíbrio e marcha em pacientes com DP.

\section{MÉTODO}

Realizou-se um estudo intervencional no Ambulatório de Fisioterapia do Hospital de Base (HB) da Fundação Faculdade Regional de Medicina (FUNFARME), na cidade de São José do Rio Preto (SP), Brasil, no período de setembro de 2017 a janeiro de 2018. Esta pesquisa foi submetida ao Comitê de Ética em Pesquisada Faculdade de Medicina de São José do Rio Preto/SP- FAMERP, sendo aprovada sob protocolo CAAE 78108017.7.0000.5415.

A amostra foi composta por indivíduos, de ambos os sexos, que apresentavam DP diagnosticada por um médico neurologista. Como critério de inclusão, os indivíduos deveriam ser classificados em estágio I a III da doença, pontuados pela escala de Hoehn \& Yahr. ${ }^{11}$ E como critério de exclusão os indivíduos não poderiam ser portadores de doenças reumáticas verificadas em questionário durante avaliação inicial e deveriam ter nível cognitivo preservado.

As coletas de dados foram realizadas no ginásio de atendimento do Ambulatório de Fisioterapia do Hospital de Base (HB), de São José do Rio Preto (SP). Todos os participantes foram comunicados quanto ao objetivo e aos procedimentos do estudo e assinaram um termo de consentimento livre e esclarecido. O estudo seguiu as diretrizes e os princípios éticos das pesquisas envolvendo seres humanos presentes na Resolução no 196/96, do Conselho Nacional de Saúde.

O protocolo proposto foi iniciado com uma avaliação inicial com coleta de dados pessoais, história clínica, bem como aplicação das escalas de Hoehn \& Yahr e de equilíbrio e marcha de Tinetti. Os indivíduos então foram orientados quanto ao programa de reabilitação e à importância de comparecerem em todos os dias do estudo. A conduta fisioterapêutica foi aplicada 2 vezes por semana, com duração de 45 minutos cada sessão, em um período de 8 semanas totalizando 16 sessões.

O programa de reabilitação do equilíbrio e marcha foi composto da seguinte forma:

- Alongamentos ativos de membros superiores e inferiores, tronco e coluna vertebral, em posição sentada, em cadeira com pés apoiados na bola suíça, usando faixa elástica;

- Manipulação passiva das cadeias musculares, articulares e coluna vertebral, em movimentos funcionais no tablado;

- Cinesioterapia ativa por meio da propriocepção em decúbito dorsal, sentado e em posição ortostática, utilizando BosuBall ${ }^{\circledR}$ e tábua lateral proprioceptiva;

- Cinesioterapia ativa estática por meio da dissociação de cinturas escapular e pélvica, em decúbito dorsal, sentado e em posição ortostática de frente para o espaldar, alternando os membros;

- Cinesioterapia dinâmica em posição ortostática, por meio da dissociação das cinturas escapular e pélvica, com paciente posicionado na barra paralela, utilizando rolo de posicionamento;

- Treino ativo de marcha e equilíbrio dinâmico e automático em barra paralela.

As condutas foram realizadas nessa ordem, em todas as 16 sessões, com todos os pacientes individualmente, no ginásio do Ambulatório de Fisioterapia do HB. Utilizaram-se como equipamentos para o programa de reabilitação: bola suíça, faixa elástica, tablado simples, BosuBall ${ }^{\circledR}$, tábua lateral proprioceptiva, espaldar, rolo de posicionamento e barras paralelas. Realizou-se a reavaliação, com a reaplicação da escala de Tinetti, na $17^{\mathrm{a}}$ sessão, para posterior análise dos dados.

Devido à diversidade de sinais e sintomas, a DP atinge os pacientes de vários modos. Os sintomas podem ser leves, graves, ocorrer com frequência ou esporadicamente. Devido a essa diversidade, ao longo do tempo, desenvolveram-se es- 
calas para avaliar e monitorar a evolução da doença e a eficácia de tratamento. ${ }^{7}$ Dentre várias escalas existentes, a de Hoehn \& Yahr foi escolhida para este estudo por ser amplamente utilizada, ser uma ferramenta de classificação simples e oferecer alguns pontos de referência para ajudar a determinar o estágio da doença do paciente, com base nos sintomas motores. Por meio dessa escala, a DP pode ser classificada em cinco estágios:

1. unilateral;

2. bilateral;

3. instabilidade postural moderada;

4. instabilidade postural grave;

5. locomoção dependente.

O tempo gasto em cada estágio de evolução da doença varia de paciente para paciente ${ }^{11}$.

Desenvolvida por Tinetti, em 1986, e validada no Brasil por Gomes, em 2003, a escala de Tinetti é usada para avaliar equilíbrio e marcha. ${ }^{12}$ Consiste em uma escala de 16 tarefas que são avaliadas por meio da observação do examinador. A cada tarefa, a resposta pode ser classificada como:

- normal: 0 ;

- $\quad$ adaptável: 1 ;

- $\quad$ anormal: 2 .

São atribuídos pontos de 0-2 na realização das tarefas, totalizando, no máximo, 48 pontos. Os valores abaixo de 19 pontos e entre 19 e 24 pontos representam, respectivamente, alto e moderado risco de quedas. ${ }^{13}$

O índice de Tinetti é compreendido por duas escalas: de equilíbrio e de marcha. A primeira é composta de nove itens e a segunda, de sete. Nas tarefas/manobras em que é necessário o uso de uma cadeira, o paciente inicia a avaliação em uma cadeira rígida, sem braços e de costas eretas. Para definir o escore de equilíbrio e a marcha, a escala de Tinetti é um bom instrumento, uma vez que detecta alterações na locomoção, diagnostica e quantifica a gravidade do comprometimento e prediz o risco de quedas. ${ }^{12,13}$

Ao final do protocolo experimental, os dados coletados foram analisados estatisticamente. Para isso, realizou-se estatística descritiva e os dados foram apresentados em valores de média e desvio-padrão. Teste de Shapiro-Wilk foi aplicado para analisar a normalidade de distribuição dos dados. Estatística inferencial foi aplicada por meio do teste $t$ pareado de Student, para comparar os momentos antes e após a intervenção. As análises foram realizadas no programa Statistical Package for the Social Sciences, versão 13.0 para Windows, sendo considerados estatisticamente significativos valores $\mathrm{p} \leq 0,05$.

\section{RESULTADOS}

Participaram do estudo 10 indivíduos, $60 \%$ do sexo masculino, com média de idade $66,6 \pm 10,7$ anos. A caracterização dos indivíduos participantes do estudo está disposta na Tabela 1.

Observou-se aumento significativo para os itens levanta-se da cadeira $(p=0,05)$ e desequilíbrio no externo $(\mathrm{p}=0,01)$ do teste de equilíbrio da escala de Tinetti, na comparação entre os períodos antes e após a intervenção fisioterapêutica (Tabela 2).

Verificou-se aumento significativo no item comprimento e altura do passo $(\mathrm{p}=0,005)$ e no escore total $(\mathrm{p}=0,05)$ do teste de marcha da escala de Tinetti, na comparação entre antes e após a intervenção fisioterapêutica (Tabela 3).

Na comparação do escore total da escala de Tinetti, observou-se incremento significativo $(p=0,04)$ entre os momentos antes e após a intervenção fisioterapêutica (Tabela 4).

\section{DISCUSSÃO}

O presente estudo demonstrou aumento significativo para os itens levanta-se da cadeira e desequilíbrio no externo do teste de equilíbrio da escala de Tinetti, na comparação entre an-

Tabela 1. Caracterização dos indivíduos com doença de Parkinson participantes do estudo.

\begin{tabular}{|lccccc} 
Indivíduo & Sexo & Idade (anos) & Tempo diagnóstico (anos) & Hoehn \& Yahr & Medicações em uso regular \\
\hline 1 & M & 80 & 15 & 3 & Prolopa \\
\hline 2 & M & 66 & 8 & 1,5 & Levodopa, Prolopa \\
3 & F & 59 & 4 & 1,5 & Levodopa e Fluoxetina \\
\hline 4 & F & 80 & 4 & 2,5 & Prolopa, Metformina, Sivastatina \\
\hline 5 & M & 44 & 11 & 1,5 & Prolopa, Levodopa, Pramipexol \\
\hline 6 & M & 73 & 15 & 3 & Prolopa, Pramipexol, Amantadina \\
\hline 7 & M & 70 & 14 & 2,5 & Prolopa, Artani \\
\hline 8 & M & 67 & 7 & 2 & Prolopa, Losartana \\
\hline 9 & F & 68 & 11 & 2,5 & Prolopa \\
\hline 10 & F & 59 & 4 & 1 & Levodopa \\
\hline Média \pm DP & - & $66,6 \pm 10,7$ & $9,3 \pm 4,5$ & $2,1 \pm 0,6$ & - \\
\hline
\end{tabular}

M: masculino; F: feminino; DP: desvio padrão. 
tes e após a intervenção fisioterapêutica, também verificou-se aumento significativo no item comprimento e altura do passo e no escore total do teste de marcha. Na comparação do escore total da escala observou-se incremento significativo entre os momentos antes e após a intervenção fisioterapêutica realizada.

Sousa e Bezerra ${ }^{14}$ verificaram a efetividade do treinamento de realidade virtual na marcha em pacientes com DP e seus resultados evidenciaram redução do tempo para percorrer o trajeto, aumento da cadência após o treino em relação às avaliações iniciais e redução do número de erros de colocação do pé no alvo após o treino. A intervenção foi efetiva

Tabela 2. Análise das variáveis do teste de equilíbrio antes e após intervenção fisioterapêutica.

\begin{tabular}{lccc} 
& Antes & Após & Valor p \\
\hline Equilíbrio sentado & $0,7 \pm 0,4$ & $0,8 \pm 0,4$ & 0,34 \\
\hline $\begin{array}{l}\text { Levanta-se da cadeira } \\
\text { Tentativas para se } \\
\text { levantar }\end{array}$ & $1,3 \pm 0,67$ & $1,8 \pm 0,42$ & $\mathbf{0 , 0 5 *}$ \\
\hline $\begin{array}{l}\text { Equilíbrio de pé } \\
\text { imediato }\end{array}$ & $1,5 \pm 0,54$ & $1,7 \pm 0,48$ & 0,34 \\
\hline Equilíbrio de pé & $1,7 \pm 0,48$ & $1,7 \pm 0,48$ & $>0,99$ \\
\hline $\begin{array}{l}\text { Desequilíbrio no } \\
\text { esterno }\end{array}$ & $1,4 \pm 0,51$ & $1,9 \pm 0,31$ & $\mathbf{0 , 0 1 *}$ \\
\hline Olhos fechados & $0,8 \pm 0,4$ & $0,9 \pm 0,3$ & 0,34 \\
\hline Girar 360 & $1,4 \pm 0,51$ & $1,4 \pm 0,51$ & $>0,99$ \\
\hline Sentar-se & $1,5 \pm 0,52$ & $1,4 \pm 0,51$ & 0,34 \\
\hline Total & $11,7 \pm 4,13$ & $12,8 \pm 3,29$ & 0,11 \\
\hline
\end{tabular}

*Teste $t$ pareado.

Tabela 3. Análise das variáveis do teste de marcha antes e após intervenção fisioterapêutica.

\begin{tabular}{lccc} 
& Antes & Após & Valor p* \\
\hline Iniciação da marcha & $0,7 \pm 0,4$ & $0,7 \pm 0,4$ & $>0,99$ \\
\hline $\begin{array}{l}\text { Comprimento e altura } \\
\text { do passo }\end{array}$ & $0,5 \pm 0,7$ & $1,1 \pm 0,5$ & $\mathbf{0 , 0 0 5}$ \\
\hline Simetria do passo & $0,6 \pm 0,5$ & $0,8 \pm 0,4$ & 0,16 \\
\hline Continuidade do passo & $0,7 \pm 0,4$ & $0,9 \pm 0,3$ & 0,16 \\
\hline Desvio da linha reta & $1,5 \pm 0,5$ & $1,2 \pm 0,4$ & 0,19 \\
\hline Tronco & $1,4 \pm 0,5$ & $1,2 \pm 0,7$ & 0,44 \\
\hline Base de apoio & $0,7 \pm 0,4$ & $0,7 \pm 0,4$ & $>0,99$ \\
Total & $6,9 \pm 2,8$ & $11,2 \pm 7,7$ & $\mathbf{0 , 0 5}$ \\
\hline
\end{tabular}

*Teste $t$ pareado.

Tabela 4. Escore total da escala Tinetti antes e após a intervenção fisioterapêutica.

$\begin{array}{cccc} & \text { Antes } & \text { Após } & \text { Valor p* } \\ \text { Escore total } & 18,6 \pm 6,8 & 20,8 \pm 5,8 & \mathbf{0 , 0 4}\end{array}$

*Teste $t$ pareado. principalmente em reduzir o tempo para percorrer o percurso e corrigir erros de colocação do pé no alvo. Os achados desses autores são similares aos encontrados no presente estudo quanto à melhora do comprimento e da altura do passo e ao escore total da marcha da escala de Tinetti. Contudo, é importante ressaltar os diferentes métodos de intervenção utilizados entre os estudos.

Outros autores verificaram a efetividade da prática em grupo da fisioterapia associada à yoga e à musicoterapia nas variáveis cognição, equilíbrio, mobilidade e independência funcional de pessoas com DP. O programa de reabilitação associando as intervenções realizadas em grupo e com frequência semanal única foi efetivo para melhorar todas as variáveis estudadas. ${ }^{15}$ Mais uma vez, pode-se observar similaridade entre o trabalho citado e o presente estudo, dessa vez na melhora do equilíbrio dos indivíduos portadores da DP após intervenção fisioterapêutica, porém, novamente, verificou-se diferença nos protocolos de intervenção aplicados.

Uma metanálise de ensaios clínicos randomizados ${ }^{16}$ evidenciou que programas de reabilitação com exercícios específicos para a marcha melhoram o equilíbrio, a mobilidade funcional e os parâmetros velocidade, comprimento do passo e passada da marcha por combinar estratégias motoras e cognitivas em indivíduos com DP. Essas evidências confirmam a importância e a necessidade de programas de reabilitação focados no equilíbrio e na marcha em pacientes com DP, visto que aumentam a independência funcional e melhoram a qualidade de vida desses pacientes.

Yamashita et al. ${ }^{17}$ demonstraram que um protocolo fisioterapêutico associando cinesioterapia e musicoterapia foi eficaz nos desfechos equilíbrio e marcha em pessoas com DP. Outros autores também encontraram resultados satisfatórios para velocidade, comprimento e tempo do passo e passada e cadência da marcha em tarefa única e dupla tarefa por meio de um programa de reabilitação que incluía caminhada ouvindo música. ${ }^{18}$

Os déficits motores são os principais acometimentos da DP, sendo a marcha prejudicada nesses pacientes, segundo a literatura, principalmente em situações de realização simultânea de outras tarefas. ${ }^{19}$ Tais alterações podem ser explicadas por déficits cognitivos, visto que esses pacientes apresentam diminuição na velocidade de processamento, memória de trabalho, função executiva e habilidades de atenção. Morris et al..$^{20}$ já demonstraram que intervenções fisioterapêuticas cognitivo-motoras e prática regular de exercícios favorecem ajustes na velocidade da marcha e no equilíbrio, bem como promovem melhor desempenho de tarefas associadas durante a marcha em pacientes com DP.

$\mathrm{Na}$ avaliação do equilíbrio e da marcha pela escala de Tinetti, verificou-se que nem todos os índices obtiveram diferença significativa, porém, quando observado o escore total, houve melhora geral em todos os aspectos avaliados, o que pode ser explicado pela intervenção ter como recurso a plasticidade neural, ou seja, a capacidade que o sistema nervoso central (SNC) tem de modificar algumas das suas propriedades morfológicas e funcionais em resposta às alterações do ambiente. Na presença de lesões, o SNC utiliza-se dessa 
capacidade na tentativa de recuperar funções perdidas e/ou fortalecer funções similares relacionadas às originais. ${ }^{8,9}$

Força e resistência musculares, flexibilidade e agilidade são componentes relacionados com o equilíbrio e a marcha, havendo evidências na literatura sobre correlação positiva entre força muscular e tempo de execução de atividades motoras. Esses componentes interagem para o desempenho das atividades motoras cotidianas, como sentar-se, fica de pé e deambular. ${ }^{15}$ Pacientes com DP apresentam prejuízos no automatismo devido ao comprometimento estrutural dos núcleos da base, assim, um controle consciente constante torna-se necessário para a marcha. ${ }^{21}$

Um fator que pode ser ressaltado como característica peculiar do presente estudo é o conhecimento do paciente acerca de cada exercício proposto e sobre os objetivos a serem atingidos, o que lhes proporcionou perceber a importância de realizar de maneira correta e funcional cada fase de cada exercício, colaborando com o tratamento e conhecendo melhor suas dificuldades e facilidades motoras no decorrer do tempo.

Sabe-se não há cura para a $\mathrm{DP}$, mas um programa fisioterapêutico adequado junto ao acompanhamento médico e ao tratamento farmacológico adequado de cada paciente é de grande importância como forma de retardar a progressão dos sintomas e proporcionar melhor qualidade de vida a esses doentes. ${ }^{10} \mathrm{~A}$ intervenção realizada buscou diminuir a disfunção física e permitir ao indivíduo realizar atividades de seu dia a dia com maior eficiência e independência possível.

A proposta de realização do programa de reabilitação trabalhou de forma progressiva as limitações de forma global e periódica, a fim de alcançar a melhora do equilíbrio e da marcha. Todos os pacientes do estudo já realizavam fisioterapia convencional, porém nenhuma das condutas baseava-se na progressividade e nas repetições, ou seja, não contemplavam a plasticidade neural. Havia dificuldade de realização devido ao esquecimento dos exercícios de uma sessão para outra.

Não houve controle quanto à realização dos exercícios domiciliares, não foi quantificado o tempo em que esses índividuos começaram a realizar a fisioterapia convencional, o tamanho amostral reduzido e a falta de um grupo controle para comparação dos efeitos do programa de reabilitação proposto são fatores que podem ser destacados como limitações do estudo.

Fundamental destacar que a cooperação dos pacientes contribuiu para a realização dos exercícios e resultados. De forma geral, o empenho na realização dos exercícios e o comprometimento com o estudo foram indispensáveis. Para melhora dos resultados, poderiam ser empregados maior tempo de intervenção, maior número de pacientes e adequação à progressão de alguns exercícios com carga ou resistência, para controle de massa muscular e diminuição dos efeitos da sarcopenia. ${ }^{11}$

\section{CONCLUSÃO}

O programa de reabilitação proposto demonstrou melhoras significativas nos movimentos que envolvem tarefas do cotidiano, como levantar-se de uma cadeira e comprimento e altura do passo durante a deambulação, componentes indis- pensáveis para equilíbrio e marcha adequados em um grupo de pacientes com DP.

\section{REFERÊNCIAS}

1. Silva DCL, Vianna E, Martins CP, Martins JV, Rodrigues EC, Oliveira LAS. Perfil dos indivíduos com doença de Parkinson atendidos no setor de fisioterapia de um hospital universitário no Rio de Janeiro. Rev Bras Neurol. 2015;51(4):100-5.

2. Souza PI, Santos LM, Santana VS, Feitosa AG. Capacidade funcional de idosos com doença de Alzheimer e Parkinson: revisão bibliográfica. Rev Pesq Fisioter. 2014;4(1):78-84. http://dx.doi. org/10.17267/2238-2704rpf.v4i1.377

3. Choi YI, Song CS, Chun BY. Activities of daily living and manual hand dexterity in persons with idiopathic Parkinson. J Phys Ther Sci. 2017;29(3):457-60. http:// dx.doi.org/10.1589/jpts.29.457

4. Martin DM, Samà A, López CP, Català A, Arostegui JMM, Cabestany J, et al. Home detection offreezing of gait using support vector machine through a single waist-worn triaxial accelerometer. Plos One. 2017;12(2):e0171764. https://doi.org/10.1371/journal. pone. 0171764

5. Scalzo PL, Santos RMS, Carvalho DV, Magalhães HC, Christo PP, Souza MS, et al. Caracterização da dor em pacientes com doença de Parkinson. Rev Bras Neurol. 2018;54(4):19-25.

6. Fuxe K, Marcellino D, Genedani S, Agnati L. Adenosine A2A receptors, dopamine $\mathrm{D} 2$ receptors and their interactions in Parkinson's disease. Mov Disord. 2007;22(14):19902017. https://doi.org/10.1002/mds.21440

7. Duchesne C, Gheysen F, Bore A, Albouy G, Nadeau A, Robillard ME, et al. Influence of aerobic exercise training on the neural correlates of motor learning in Parkinson's disease individuals. Neuroimage Clin. 2016;12:559-69. http://dx.doi.org/10.1016/j.nicl.2016.09.011

8. Goulart F, Santos CC, Salmela LFT, Cardoso F. Análise do desempenho funcional em pacientes portadores de doença de Parkinson. Acta Fisiatr. 2004;11(1):12-6. https://doi.org/10.5935/0104-7795.20040001

9. Carvalho A, Barbirato D, Araujo N, Martins JV, Cavalcante JLS, Santos TM, et al. Comparison of strength training, aerobic training, and additional physical therapy as supplementary treatments for Parkinson's disease: pilot study. Clin Interv Aging. 2015;2015(10):183-91. http://dx.doi.org/10.2147/CIA.S68779

10. Roeder L, Costello JT, Smith SS, Stewart IB, Kerr GK. Effects of resistance training on measures of muscular strength in people with Parkinson's disease: a systematic review and meta-analysis. Plos One. 2015;10(7):e0132135. http://dx.doi.org/10.1371/ journal.pone.0132135

11. Mello MPB, Botelho ACG. Correlação das escalas de avaliação utilizadas na doença de Parkinson com 
aplicabilidade na fisioterapia. Fisioter Mov. 2010;23(1):1217. https://doi.org/10.1590/S0103-51502010000100012

12. Gomes GS. Tradução, adaptação transcultural e exame das propriedades de medida da escala "PerformanceOrientes Mobility Assessment" (POMA) para uma amostra de idosos brasileiros institucionalizados [dissertação]. Campinas: Universidade Estadual de Campinas; 2003.

13. Karuka AH, Silva JAMG, Navega MT. Análise da concordância entre instrumentos de avaliação do equilíbrio corporal em idosos. Rev Bras Fisioter. 2011;15(6):460-6. http://dx.doi.org/10.1590/S141335552011000600006

14. Sousa ASK, Bezerra PP. A realidade virtual por meio do tapete de vídeodança melhora a marcha de pacientes com doença de Parkinson. Rev Bras Neurol. 2016;52(1):21-9.

15. Sousa ASK, Lopes KL, Fragnani SG, Nery T, Werner E, Bezerra PP. Fisioterapia associada a yoga e musicoterapia na doença de Parkinson: ensaio clínico. Rev Bras Neurol. 2017;53(3):31-40.

16. de Dreu MJ, van der Wilk AS, Poppe E, Kwakkel G, van Wegen EE. Rehabilitation, exercise therapy and music in patients with Parkinson's disease: a metaanalysis of the effects of music-based movement therapy on walking ability, balance and quality of life. Parkinsonism Relat Disord. 2012;18(Suppl. 1):S114-9. http://dx.doi.org/10.1016/S1353-8020(11)70036-0
17. Yamashita FC, Saito TC, Almeida IA, Barboza NM, Santos SMS. Efetividade da fisioterapia associada a musicoterapia na doença de Parkinson. ConSc Saúde. 2012;11(4):677-84. https://doi.org/10.5585/conssaude. v11n4.3857

18. de Bruin N, Doan JB, Turnbull G, Suchowersky O, Bonfield S, Hu B, et al. Walking with music is a safe and viable tool for gait training in Parkinson's disease: the effect of a 13-week feasibility study on single and dual task walking. Parkinsons Dis. 2010;483530. http:// dx.doi.org/10.4061/2010/483530

19. Pacchetti C, Mancini F, Aglieri R, Fundarò C, Martignoni E, Nappi G. Active music therapy in Parkinson's disease: na integrative method for motor and emotional rehabilitation. Psychosom Med. 2000;62(3):386-93. https://doi. org/10.1097/00006842-200005000-00012

20. Morris S, Morris ME, Iansek R. Reliability of measurements obtained with the Timed "Up e Go" Test in people with Parkinson disease. Phys Therapy. 2001;81(2):810-8. https://doi.org/10.1093/ ptj/81.2.810

21. Brauer SG, Woollacott MH, Lamont R, Clewett S, O'Sullivan J, Silburn P, et al. Single and dual task gait training in people with Parkinson's disease: a protocol for a randomised controlled trial. BMC Neurol. 2011;11:90. http://dx.doi.org/10.1186/14712377-11-90

\section{Como citar este artigo:}

Dantas VM, Guiduce RO, Foss MHD, Ferreira LL. Programa de reabilitação de equilíbrio e marcha em pacientes com doença de Parkinson. Rev Fac Ciênc Méd Sorocaba. 2020;22(3):93-8. https://doi.org/10.23925/1984-4840.2020v22i3a2 